\title{
Use and Misuse of Antibiotics
}

Md. Nurul Amin ${ }^{1}$

During the last two decades the volume of pharmaceuticals production has increased enormously, although, their availability and rational use remain a problem for much of the world's population. This is particularly so in case of antibiotic uses. These problems derive from a combination of financial and budgetary constraints, market inefficiencies and distortions, and behaviour of health systems, prescribers, dispensers, consumers and pharmaceutical companies. ${ }^{1}$ Nearly one-thrid to two-thirds of primary health care patients are found to receive antibiotics - perhaps twice what is clinically needed. ${ }^{2}$ The rational use of drugs requires that patients receive medications appropriate to their clinical needs, in doses that meet their own individual requirements for an adequate period of time, and at the lowest cost to them and their community. ${ }^{1}$ In developing countries, acquired bacterial resistance to antimicrobial agents is common in isolates from healthy persons and from persons with community-acquired infections. Complex socioeconomic and behavioral factors associated with antibiotic resistance include misuse of antibiotics by health professionals, unskilled practitioners and public in general. Poor drug quality and inadequate surveillance also contribute to emergence and spread of antibiotic-resistant bacteria. ${ }^{3}$

Tunger et $\mathrm{al}^{4}$ in a study on 937 inpatients demonstrated that $16.6 \%$ of the patients received antibiotics. Of them $63.5,23.0$ and $13.5 \%$ received a single, double and three agents respectively. The prophylactic antibiotic use was $23.9 \%$, empiric use $71.4 \%$ and therapeutic culture-based use $4.7 \%$. The rational use was $45.7 \%$ and it was statistically higher in those patients from whom specimens had been taken for culture than in patients receiving prophylactic or empiric antibiotics. In medical wards, rational antibiotic usage was $55.1 \%$, while it was $26.3 \%$ in surgical wards.

More than two decades ago Rashid and colleagues ${ }^{5}$ in a survey of the use of antibiotics in seven community hospitals of Bangladesh demonstrated that $62 \%$ of patients were given antibiotics without any evidences of infection. The trend has not changed yet; rather it has further deteriorated as is evidenced by the following data.

Varies and colleagues ${ }^{6}$ in a study of prescribing behaviour of physicians in Bangladesh in 1994 showed that around $50 \%$ of the antibiotics prescribed were irrational in terms of indications, dose and duration. More recent studies show that prophylactic uses of antimicrobials in different categories of surgeries are irrational in general, which imposes an enormous burden on the patients or the system in terms of cost. In a study conducted by Islam et $\mathrm{al}^{7}$ in 2007 , the average duration of stay in hospital and average cost of antimicrobial therapy for patients of caesarean section admitted in Bangabandhu Sheikh Mujib Medical University (BSMMU) were 8.6 days and 1693 Taka respectively. After intervention with 'Prescription Audit \& Feedback', the expensive treatment plan reduced from $93.3 \%$ to $0 \%$ and the average hospital stay and cost for antimicrobial therapy reduced to 7.6 days and 511 Taka respectively. Rahman et $\mathrm{al}^{8}$ published a study in 2009 in which 600 prescriptions of private practitioners were analysed using WHO/INRUD indicators. Nearly three-quarter (72.5\%) of these prescriptions contained at least one antibiotic, though many of them did not have any indications (written on the prescriptions) for which the antibiotics were prescribed. Thus the data of antibiotics use from 1986 to 2009 indicate that 
the problem has worsened over time. Though more recent data are not in hand, the picture seems to be even worse.

Generalized presence of irrationalities in prescribing antibiotics indicates that traditional teaching in medical colleges does not adequately prepare students for rational therapeutics. This is perhaps, so because their pharmacology training has concentrated more on theory than on practical aspects of prescribing. Prescribing behaviour of the medical graduates depends on how and what they have taught and trained about drugs during their undergraduate course. ${ }^{9}$

The emergence of antibiotic resistant bacteria is a major problem throughout the world and rational use of antibiotics is, therefore, very important. That purpose the World Health Organization (WHO) has developed and validated several indicators to provide an appropriate means to assess a country's drug use patterns and to measure the efficacy of interventions. The indicators are highly standardized and are recommended for inclusion in studies on drug use. They provide a simple tool for quickly and reliably assessing a number of critical aspects of pharmaceutical use in primary health care. ${ }^{10-12}$

The indicators are used to measure performance in three areas related to the rational use of drugs in primary health care facilities: prescribing practices, patient care and facility standards. In this respect, five core prescribing indicators have been developed and standardized (WHO/INRUD). These include:

- average number of drugs prescribed per encounter

- percentage of drugs prescribed by generic name

- percentage of encounters where antibiotics are prescribed

- percentage of encounters where injections are prescribed, and

- percentage of drugs prescribed from the essential drugs list (EDL).

These indicators provide basic information concerning drug prescribing practices. The information obtained can be used to determine implementation priorities in the drugs sector, and to assess the effectiveness of overall drugs policies irrespective of the political, economic or social (including health status) conditions in a country. ${ }^{10-13}$

Similarly, the Centre for Disease Control, Atlanta has outlined principles of antibiotic use which are as follows: ${ }^{14}$

1. Use antibiotics only where the benefits are scientifically demonstrated and substantial.

2. Use the narrowest spectrum of antimicrobial (unless otherwise indicated) to treat known or likely pathogens.

3. Use a single drug unless it has been proven that combination therapy is required to ensure efficacy or reduce the selection of clinically significant resistance.

4. Use a dose that is high enough to ensure efficacy and low enough to minimize the risk of adverse events.

5. Therapy should be directed based on culture and susceptibility results.

6. Duration should be as short as possible and should not exceed $>7$ days unless there is proof that this duration is inadequate. ${ }^{15}$

Antibiotics are one of the most commonly prescribed drugs in the field of medical practice. Although they are life-saving, they act as doubleedged sword. If prescribed appropriately they can save life, but if prescribed inappropriately they may cause more harm than good. Inappropriate use of antimicrobials is observed throughout the world leading to emergence of drug-resistant bacteria, drug toxicity and increased health care costs. If we do not take immediate measure with due importance to avert the existing situation, perhaps very few antibiotics will remain functioning in near future. To get rid of these problems, rational use of drugs, particularly antibiotics should be ensured in every tire of our health care settings. However, as multiple factors are associated with irrational prescribing of antibiotics, strict antibiotic policy is deemed essential, which, in turn, requires detailed information about existing antibiotic-prescribing behaviour of physicians. 


\section{References}

1. WHO Action Programme on Essential Drugs. Report of the WHO Expert Committee on National Drug Policies. Geneva, World Health Organization, 1995.

2. Quick et al. 1997, 'Managing drug supply',

3. Okeke IN, Lamikanra A and Edelman OR. Socioeconomic and behavioral factors leading to acquired bacterial resistance to antibiotics in developing countries. Emerg Infect Dis 1999; 5(1): 18-27.

4. Tunger O, Dinç G, Ozbakkaloglu B, Atman UC, Algun U. Evaluation of rational antibiotic use. Int J Antimicrob Agents 2000; 15(2): 131-5.

5. Rashid HU, Chowdhury SAR, Islam N. Pattern of antibiotic use in two teaching hospitals. Trop Doct 1986; 18(4): 152-4.

6. De Varies TPGM, Henning RH, Hogerzeil HV, Fresle DA. Guide to good prescribing: A practical manual. WHO/DAP 1994; 94: 11.

7. Islam MS, Rahman MS, Misbahuddin M. Impact of prescription audit \& feedback' on pattern of prophylactic antimicrobials in caesarean section: A cost reduction perspective. Bangladesh J Physiol Pharmacol 2007; 23(1 \& 2): 1-9.
8. Rahman Z, Nazneen R, Begum M. Evaluation of the prescribing pattern of the private ptactioners by the undergraduate medical students. Bangladesh J Pharmacol 2009; 4: 73-5.

9. Schwartz S, Griffin T. Medical thinking: The psychology of medical judgment and rational decision-making. Springer, New York, 1986.

10. Anonymus. WHO Action Programme on Essential Drugs and Vaccines. How to investigate drug use in health facilities: selected drug use indicators. Geneva, World Health Organization, 1993.

11. Carrin G. Drugs prescribing: a discussion of its variability and (ir)rationality. Health Policy 1987; 7: 7394.

12. Venulet J. Rational prescribing of drugs. International J Clin Pharmacol \& Biopharmacy 1997; 15; 151-4.

13. Le Grand A, Hogerzeil HV, Haaijer-Ruskamp FM. Intervention research in rational use of drugs: a review. Health Policy \& Planning 1999; 14: 89-102.

14. Antibiotic/Antimicrobial Resistance. CDC Website At: http://198.246.98.21/drugresistance/index.htm.

15. Therapeutic Guidelines, Antibiotic, version 13, Australia, 2006. 